\title{
Affine Maps That Induce Polyhedral Complex Isomorphisms*
}

\author{
A. Dress, ${ }^{1}$ K. T. Huber, ${ }^{2}$ and V. Moulton ${ }^{3}$ \\ ${ }^{1}$ FSPM-Strukturbildungsprozesse, University of Bielefeld, \\ D-33501 Bielefeld, Germany \\ dress@mathematik.uni-bielefeld.de \\ ${ }^{2}$ Institute of Fundamental Sciences, Massey University, \\ Private Bag 11 222, Palmerston North, New Zealand \\ kathi@dirac.fmi.mh.se \\ ${ }^{3}$ FMI, Mid Sweden University, \\ Sundsvall S-851 70, Sweden \\ vince@dirac.fmi.mh.se
}

\begin{abstract}
In this paper we show that an affine bijection $f: T_{1} \rightarrow T_{2}$ between two polyhedral complexes $T_{1}, T_{2}$, both of which consist of a union of faces of two convex polyhedra $P_{1}$ and $P_{2}$, necessarily respects the cell-complex structure of $T_{1}$ and $T_{2}$ inherited from $P_{1}$ and $P_{2}$, respectively, provided $f$ extends to an affine map from $P_{1}$ into $P_{2}$. In addition, we present an application of this result within the area of T-theory to obtain a far-reaching generalization of previous results regarding the equivalence of two distinct constructions of the phylogenetic tree associated to "perfect" (that is, treelike) distance data.
\end{abstract}

\section{Introduction}

In this paper a polyhedron is understood to be a subset of some affine space that is the intersection of a finite collection of closed half-spaces, and a polytope is a compact polyhedron. Thus, for us, polyhedra and polytopes are all convex. Polyhedral cell complexes, in turn, are finite collections $\mathcal{C}$ of polytopes, called the cells of $\mathcal{C}$, such that (i) each face

\footnotetext{
* While this work was done, the first author worked as Distinguished Visiting Professor at the Department of Chemical Engineering, City College, CUNY, New York. The second author thanks the New Zealand Marsden Fund for its support, and the FSPM-Strukturbildungsprozesse, University of Bielefeld, for hosting her during part of the work. The third author thanks the Swedish Natural Science Research Council (NFR) for its support (Grant \# M12342-300), and the FSPM-Strukturbildungsprozesse, University of Bielefeld, for hosting him during part of the work.
} 
of a member of $\mathcal{C}$ is itself a member of $\mathcal{C}$ and (ii) the intersection of any two members of $\mathcal{C}$ is either a face of each or is empty. Such cell complexes are studied in discrete geometry. For example, they arise naturally as boundaries of polytopes or, more generally, as collections of bounded faces of polyhedra [21], and they are of use in the study of fiber polytopes [25] and in combinatorial optimization [23]. They can also be used to justify de Bruijn's dualization principle which allows us to construct zonotopal Penrose-type tilings from multigrids of hyperplanes [4] (see the Appendix for more details).

Polyhedral complexes play an important role in the emerging field of T-theory [15], too. Here, an approach to distance analysis is taken that relies on constructions that use distance data defined on some (usually finite) set $X$ as follows: First, one embeds $X$ into some affine space $A$, then one constructs some large polyhedron $P$ in $A$ that contains the image of $X$ and, finally, one considers some-generally simply connected-polyhedral subcomplex $T$ of the polyhedral complex consisting of (all or some) compact faces of $P$. Such constructions are generally motivated by the expectation that the geometric and combinatorial properties of $T$ may eventually reveal some important features of the original distance data in question.

This approach has application in subjects ranging from the theory of molecular evolution and systematic biology to psychology, archaeology, or stemmatology (see [1], [2], [6], [12], and [22]), and, provided one is willing not to care much about the finiteness restrictions above, it can be applied even in pure mathematics: Given a field $K$ and a discrete valuation $v$ of $K$ (that is, a map $v$ from $K$ into $\mathbb{Z} \cup\{-\infty\}$ satisfying the conditions $v(x)=-\infty \Longleftrightarrow x=0, v(x y)=v(x) v(y)$, and $\max (v(x), v(y)) \geq v(x+y))$ the Bruhat-Tits building associated to the general linear group $G L(K, n+1)$ of dimension $n+1$ can be identified easily with the union of all compact faces of the polyhedron $P$ that is contained in the affine space $A:=\mathbb{R}^{X}$ formed by the set of all maps $f$ from $X:=K^{n+1}-\{0\}$ into $\mathbb{R}$, and consists exactly of all those maps $f$ that satisfy the inequality

$$
v\left(\operatorname{det}\left(x_{0}, x_{1}, \ldots, x_{n}\right)\right) \leq f\left(x_{0}\right)+f\left(x_{1}\right)+\cdots+f\left(x_{n}\right)
$$

for every family of nonzero vectors $x_{0}, x_{1}, \ldots, x_{n}$ in $K^{n+1}$, while the chambers of that building coincide exactly with the maximal compact faces of $P$ (see [16] and [17]).

It was in the context of that T-theoretic approach to distance analysis described above that the following problem arose: More often than not, there are several constructions that can be applied to a given instance of distance data regarding a set $X$, and these constructions will often lead to distinct affine spaces $A, A^{\prime}, \ldots$ and distinct polyhedra $P, P^{\prime}, \ldots$, containing distinct polyhedral complexes $T, T^{\prime}, \ldots$ In such a situation, one wants to compare these complexes, and a natural way to do this is to look for affine maps between the "enveloping" affine spaces that—at least in favorable cases_-would map $P$ into $P^{\prime}$ and $T$ into $T^{\prime}$. However, even if an affine map between affine spaces would induce a bijection between certain polyhedral complexes $T, T^{\prime}$ contained in (and considered as subsets of) those affine spaces, this bijection need not induce a polyhedral-complex isomorphism, that is, a bijection between (the points in) $T$ and $T^{\prime}$ such that a subset $S$ of (points in) $T$ forms a cell in $T$ if and only if the image $S^{\prime}$ of $S$ in $T^{\prime}$ forms a cell in $T^{\prime}$.

For example, the map $f: \mathbb{R}^{2} \rightarrow \mathbb{R}:(x, y) \mapsto x-y$ induces a bijection between the polyhedral complex $P$ obtained from the two faces

$$
\{(x, 0): 0 \leq x \leq 1\} \text { and }\{(0, y): 0 \leq y \leq 1\}
$$


of the unit square $[0,1] \times[0,1]$ onto the polytope $P^{\prime}$ consisting of the interval $[-1,1]$ (which we regard as a polyhedral complex in the natural way), yet $f$ is clearly not a polyhedral-complex isomorphism.

Therefore, the following observation appeared remarkable:

(A) If the affine map induces not only a bijection between the two polyhedral complexes in question, but also maps polyhedron into polyhedron, this bijection must necessarily respect the induced cell-complex structure of the two complexes.

Although this result is quite general, it is also fairly basic and-as we shall seeits proof is quite elementary. In fact, we first expected that, to make use of it in the context of T-theory, we could just quote any appropriate textbook devoted to convex geometry. However, we searched in vain and were later assured by a number of experts that, seemingly, this result had been overlooked so far. Therefore, to highlight its basic nature within the context of elementary convex geometry, we decided not to conceal it in a highly specialized paper regarding T-constructions and Buneman complexes (where it is urgently needed-see Section 5), but to make it available to a wider audience.

The rest of the paper is organized as follows. In Sections 2 and 3 we provide some elementary results in convex geometry concerning extremal sets. We then use these to prove our main result, Theorem 1, that is stated purely in terms of convex sets, but from which the above stated result (A) on polyhedral complexes follows as an easy consequence. Finally, in the last section, we give a detailed account of the application of our main result within the context of T-theory referred to above and, in the Appendix, we recall briefly how polyhedral complexes can be used to establish de Bruijn's dualization principle.

\section{Extremal Sets}

Let $V$ be a finite-dimensional $\mathbb{R}$-vector space and let $P \subseteq V$ be some subset of $V$. A point $p \in P$ is called an extremal point of $P$ if $0<\alpha<1, x, y \in P$, and $p=\alpha x+(1-\alpha) y$ implies $x=p=y$. More generally, we define a subset $T \subseteq P$ to be an extremal subset of $P$ if, for any $u, v \in P$ and any positive real numbers $\alpha, \beta>0$ with $\alpha+\beta=1$, the assumption $\alpha u+\beta v \in T$ implies $u, v \in T$ (see p. 181 of [24]).

Clearly, any extremal point of $P$ gives rise to an extremal subset of $P$ that consists of just that single point. Furthermore, it is easy to see that $P$ itself is an extremal subset of $P$, and also that the intersection of any collection of extremal subsets of $P$ is an extremal subset of $P$, too. Motivated by this last observation, we define the extremal hull $[x]=[x]_{P}$ of any element $x \in P$ to be the intersection of all extremal subsets of $P$ that contain $x$.

Clearly, a subset $T$ of $P$ is extremal if and only if $T$ is a union of extremal subsets if and only if $T$ coincides with $\bigcup_{x \in Q}[x]$ for some set $Q \subseteq P$ if and only if $T$ coincides with $\bigcup_{x \in T}[x]$. In addition, we have:

Lemma 1. If $P \subseteq V$ is a convex set and $x \in P$, then:

(i) $[x]=\{b \in P$ : there exists $\rho>0$ with $(1+\rho) x-\rho b \in P\}$.

(ii) $[x]$ is convex. 


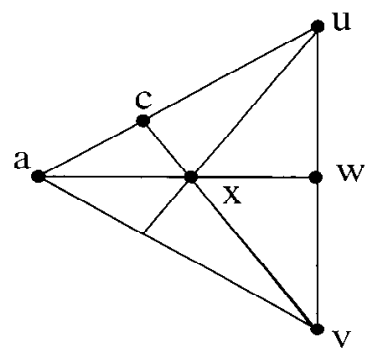

Fig. 1. The configuration considered in the proof of Lemma 1(i).

Proof. (i) Put $B:=\{b \in P$ : there exists $\rho>0$ with $(1+\rho) x-\rho b \in P\}$. Take some element $b \in B$. Then, by definition, $b \in P$ and there exists some positive real number $\rho>0$ with $b^{\prime}:=(1+\rho) x-\rho b \in P$. Hence

$$
\frac{1}{1+\rho} b^{\prime}+\frac{\rho}{1+\rho} b=x \in[x] .
$$

Since $[x]$ is an extremal set, we have $b \in[x]$ and, therefore, $B \subseteq[x]$.

Conversely, note that we trivially have $x \in B$. Thus, if we can show that $B$ is an extremal set in $P$, then $[x] \subseteq B$ follows, since $[x]$ is the minimal extremal set containing $x$. To this end, choose $u, v \in P$ and $0<\alpha<1$ with $w:=\alpha u+(1-\alpha) v \in B$ (see Fig. 1).

We claim that $u$ and $v$ are contained in $B$. By definition, there exists some positive real number $\rho>0$ with $a:=(1+\rho) x-\rho w \in P$. Thus,

$$
\begin{aligned}
c & :=\frac{1}{1+\rho \alpha} a+\frac{\rho \alpha}{1+\rho \alpha} u \\
& =\frac{1}{1+\rho \alpha}((1+\rho) x-\rho(\alpha u+(1-\alpha) v))+\frac{\rho \alpha}{1+\rho \alpha} u \\
& =\frac{1+\rho}{1+\rho \alpha} x-\frac{\rho-\rho \alpha}{1+\rho \alpha} v \\
& =\left(1+\frac{\rho(1-\alpha)}{1+\rho \alpha}\right) x-\frac{\rho(1-\alpha)}{1+\rho \alpha} v
\end{aligned}
$$

As $a, u \in P, 1 /(1+\rho \alpha), \rho \alpha /(1+\rho \alpha)>0$, and $1 /(1+\rho \alpha)+\rho \alpha /(1+\rho \alpha)=1$, we have $c \in P$, and, hence, $v \in B$ in view of $\rho(1-\alpha) /(1+\rho \alpha)>0$. Similarly, we get $u \in B$. So, $B$ is indeed extremal.

(ii) Suppose $u, v \in[x], 0<\alpha<1$, and $\beta:=1-\alpha$. In view of (i), there exist some positive real numbers $\rho, \sigma>0$ such that $u^{\prime}:=(1+\rho) x-\rho u$ and $v^{\prime}:=(1+\sigma) x-\sigma v$ are elements of $P$ (see Fig. 2).

Put $\tau:=\beta \rho+\alpha \sigma$. Then the assumption $0<\alpha<1$ immediately yields $0<\alpha \sigma / \tau<1$ and $\rho \sigma / \tau>0$. Now, the convexity of $P$ implies that

$$
w^{\prime}:=\frac{\alpha \sigma}{\tau} u^{\prime}+\frac{\beta \rho}{\tau} v^{\prime}
$$




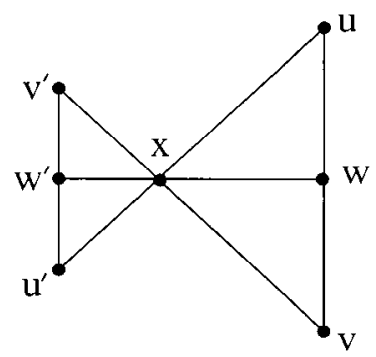

Fig. 2. The configuration considered in the proof of Lemma 1(ii).

is an element of $P$. Clearly, the convexity of $P$ also implies that $w:=\alpha u+\beta v$ is contained in $P$. Finally, we have

$$
\begin{aligned}
\left(1+\frac{\rho \sigma}{\tau}\right) x-\frac{\rho \sigma}{\tau} w & =\frac{\tau+(\alpha+\beta) \rho \sigma}{\tau} x-\frac{\rho \sigma}{\tau}(\alpha u+\beta v) \\
& =\frac{\alpha \sigma(1+\rho)+\beta \rho(1+\sigma)}{\tau} x-\frac{\alpha \sigma}{\tau} \rho u-\frac{\beta \rho}{\tau} \sigma v \\
& =\frac{\alpha \sigma}{\tau}((1+\rho) x-\rho u)+\frac{\beta \rho}{\tau}((1+\sigma) x-\sigma v) \\
& =\frac{\alpha \sigma}{\tau} u^{\prime}+\frac{\beta \rho}{\tau} v^{\prime} \\
& =w^{\prime} \in P .
\end{aligned}
$$

From this, (ii) follows immediately using (i).

\section{Extremal Sets for Polyhedra}

In this section we see that an extremal set in a polyhedron $P$ is-as one would expectjust the union of a collection of some of the faces of $P$.

So, suppose that $P$ is a polyhedron and that we are given a collection of $\mathbb{R}$-linear maps $f_{i}: V \rightarrow \mathbb{R}$ and real numbers $c_{i} \in \mathbb{R}(1 \leq i \leq n)$ with $P=\left\{x \in V: f_{i}(x) \geq c_{i}\right\}$. Clearly, the smallest face in $P$ that contains some $x \in P$ equals

$$
\text { face }(x)=\text { face }_{P}(x)=\left\{b \in P: f_{i}(x)=f_{i}(b) \text { for all } i=1, \ldots, n \text { with } f_{i}(x)=c_{i}\right\} .
$$

We now show that the extremal hull $[x]_{P}$ of a point $x$ in a polyhedron $P$ always coincides with $\operatorname{face}_{P}(x)$.

Proposition 1. Given a polyhedron $P$ and some point $x \in P$, the following subsets of $P$ coincide:

(i) $[x]$;

(ii) $\operatorname{face}(x)$;

(iii) $C(x):=\{u \in P: f(u)=f(x)$ for every linear map $f: V \rightarrow \mathbb{R}$ with $f(x)=$ inf $f(P)\}$. 
Proof. We assume that $P$ is described in terms of some linear maps $f_{i}$ and constants $c_{i}$, as discussed above, and show that "face $(x) \subseteq[x] \subseteq C:=C(x) \subseteq \operatorname{face}(x)$ " holds.

Suppose that $b \in \operatorname{face}(x)$ and that $j \in\{1, \ldots, n\}$. If either $f_{j}(x)=c_{j}$ or $f_{j}(x) \geq$ $f_{j}(b)$, then the $\mathbb{R}$-linearity of $f_{j}$ immediately implies that

$$
f_{j}((1+\rho) x-\rho b)=f_{j}(x)+\rho\left(f_{j}(x)-f_{j}(b)\right) \geq c_{j}
$$

holds for every $\rho>0$. Now, suppose that $c_{j}<f_{j}(x)<f_{j}(b)$ holds. Put

$$
\rho:=\min _{1 \leq j \leq n}\left\{\frac{f_{j}(x)-c_{j}}{f_{j}(b)-f_{j}(x)}: c_{j}<f_{j}(x)<f_{j}(b)\right\},
$$

which is clearly positive. Then the $\mathbb{R}$-linearity of $f_{j}$ together with $f_{j}(x)-f_{j}(b)<0$ yields

$$
\begin{aligned}
f_{j}((1+\rho) x-\rho b) & =f_{j}(x)+\rho\left(f_{j}(x)-f_{j}(b)\right) \\
& \geq f_{j}(x)+\frac{f_{j}(x)-c_{j}}{f_{j}(b)-f_{j}(x)}\left(f_{j}(x)-f_{j}(b)\right) \\
& =c_{j} .
\end{aligned}
$$

Thus, we immediately see that $(1+\rho) x-\rho b \in P$ and so, by Lemma 1(i), we have $b \in[x]$. Clearly, this implies face $(x) \subseteq[x]$.

Next, suppose $u \in[x]$, i.e., $u \in P$ and $v:=(1+\rho) x-\rho u \in P$ for some positive real number $\rho>0$. Let $f: V \rightarrow \mathbb{R}$ be some $\mathbb{R}$-linear map with $f(x)=\inf f(P)$. Then, in particular, $f(x) \leq f(v)$ and $f(x) \leq f(u)$. The $\mathbb{R}$-linearity of $f$ along with $v=(1+\rho) x-\rho u$ yields $f(x) \leq f(v)=(\rho+1) f(x)-\rho f(u)$ and, hence, $f(x) \geq f(u)$; thus, $u \in C$ and therefore $[x] \subseteq C$.

Now, suppose $u \in C$, and assume $c_{i}=f_{i}(x)$ for some $i \in\{1, \ldots, n\}$. Since $P$ is contained in the set of elements $y \in V$ with $f_{i}(y) \geq c_{i}$, we have

$$
c_{i}=f_{i}(x) \geq \inf f_{i}(P) \geq c_{i},
$$

that is, $f_{i}(x)=\inf f_{i}(P)$. As $u \in C$, we have $f_{i}(x)=f_{i}(u)$. Hence, $u \in$ face $(x)$ and therefore $C \subseteq \operatorname{face}(x)$.

\section{The Main Result}

In this section we prove the main result of this paper. Note that since polyhedra are convex sets, and that - as we have just seen-faces of polyhedra coincide with extremal sets of the polyhedra, statement (A) in the introduction follows directly from this result.

Theorem 1. Let $V$ and $V^{\prime}$ be finite-dimensional real vector spaces, let $P \subseteq V$ and $P^{\prime} \subseteq V^{\prime}$ be convex sets, and let $f: V^{\prime} \rightarrow V$ be some affine map with $f\left(P^{\prime}\right) \subseteq P$. Moreover, let $T$ be some extremal subset of $P$. Then the following statements hold for 
$T^{\prime}:=f^{-1}(T) \cap P^{\prime}:$

(i) $T^{\prime}$ is an extremal subset of $P^{\prime}$.

(ii) If $f$ maps $T^{\prime}$ bijectively onto $T$, then $f$ maps the smallest extremal subset $\left[x^{\prime}\right]_{P^{\prime}}$ of $P^{\prime}$ containing a given $x^{\prime} \in T^{\prime}$ bijectively onto the smallest extremal subset $\left[f\left(x^{\prime}\right)\right]_{P}$ of $P$ containing $f\left(x^{\prime}\right)$.

Proof. (i) Suppose $u, v \in P^{\prime}$ and $0<\alpha<1$ are such that

$$
w:=\alpha u+(1-\alpha) v \in T^{\prime}=f^{-1}(T) \cap P^{\prime} .
$$

The assumption that $f\left(P^{\prime}\right) \subseteq P$ along with the $\mathbb{R}$-linearity of $f$ implies $f(u), f(w) \in$ $P$ as well as $\alpha f(u)+(1-\alpha) f(v) \in T$. Since $T$ is an extremal subset of $P$, we conclude that $f(u)$ and $f(v)$ are elements in $T$. Hence, $u, v \in f^{-1}(T)$ and-as $u, v \in P^{\prime}$ by assumption-we have $u, v \in f^{-1}(T) \cap P^{\prime}=T^{\prime}$. Thus, $T^{\prime}$ is an extremal subset of $P^{\prime}$, as required.

(ii) Now, assume that the restriction $f^{\prime}:=\left.f\right|_{T^{\prime}}: T^{\prime} \rightarrow T$ of $f$ is bijective, and suppose $x^{\prime} \in T^{\prime}$ and $u^{\prime} \in\left[x^{\prime}\right]$. By Lemma 1(i), there exists some positive real number $\rho>0$ such that $(1+\rho) x^{\prime}-\rho u^{\prime} \in P^{\prime}$. Since $f\left(P^{\prime}\right) \subseteq P$ and $f$ is $\mathbb{R}$-linear, it follows that $(1+\rho) f\left(x^{\prime}\right)-\rho f\left(u^{\prime}\right) \in P$ holds. Moreover, Lemma 1(i) applied to $x:=f\left(x^{\prime}\right)$ and $b:=u:=f\left(u^{\prime}\right)$ yields $u \in[x]$. Hence, the restriction $f^{\prime \prime}:=\left.f^{\prime}\right|_{\left[x^{\prime}\right]}:\left[x^{\prime}\right] \rightarrow[x]$ is well defined.

It remains to show that $f^{\prime \prime}:\left[x^{\prime}\right] \rightarrow[x]$ is bijective. The injectivity of $f^{\prime \prime}$ follows trivially from the assumed bijectivity of $f^{\prime}$. Thus, it only remains to show that $f^{\prime \prime}$ is surjective.

So, assume $u \in[x]$, choose $\rho>0$ with $v:=(1+\rho) x-\rho u \in P$, and note that $u=\left(1+\rho^{-1}\right) x-\rho^{-1} v \in P$ implies $v \in[x]$. Choose $u^{\prime}, v^{\prime} \in T^{\prime} \subseteq P^{\prime}$ with $f\left(u^{\prime}\right)=u$ and $f\left(v^{\prime}\right)=v$, and note that

$$
x^{\prime \prime}:=\frac{\rho}{1+\rho} u^{\prime}+\frac{1}{1+\rho} v^{\prime}
$$

is in $P^{\prime}$ (because of $u^{\prime}, v^{\prime} \in P^{\prime}$ as well as $0 \leq \rho /(1+\rho), 1 /(1+\rho)$ and $\rho /(1+\rho)+$ $1 /(1+\rho)=1)$ and that

$$
f\left(x^{\prime \prime}\right)=\frac{\rho}{1+\rho} u+\frac{1}{1+\rho} v=x \in T
$$

holds. Hence, $x^{\prime \prime} \in f^{-1}(T) \cap P^{\prime}=T^{\prime}$ and $f\left(x^{\prime \prime}\right)=f\left(x^{\prime}\right)$ which, in view of our injectivity assumption, implies $x^{\prime}=x^{\prime \prime}$ and therefore $(1+\rho) x^{\prime}-\rho u^{\prime}=(1+\rho) x^{\prime \prime}-\rho u^{\prime}=$ $v^{\prime} \in P^{\prime}$. So, we must have $u^{\prime} \in\left[x^{\prime}\right]$, as required.

\section{An Application}

In [10], our main result is applied within the following context: 
Given a metric $d: X^{2} \rightarrow \mathbb{R}_{\geq 0}:(x, y) \mapsto x y:=d(x, y)$ defined on a finite set $X$, one may form Isbell's injective hull

$$
T(d):=\left\{f \in \mathbb{R}^{X}: f(x)=\max _{y \in X}(x y-f(y)) \text { for every } x \in X\right\}
$$

of the metric space $(X, d)$. It is well known that $T(d)$ is a complete metric space relative to the induced $l_{\infty}$-metric

$$
\|f, g\|_{\infty}:=\max (|f(x)-g(x)|: x \in X)
$$

( $f, g \in T(d)$ ), and that $X$ embeds canonically and isometrically into $T(d)$ via the (invariate) adjoint

$$
h: X \rightarrow T(d): x \mapsto\left(h_{x}: X \rightarrow \mathbb{R}: y \mapsto x y\right)
$$

of the bivariate map $d$. Note also that $T(d)$ —as was observed already by Isbell [19] in other notation-can be viewed as a polyhedral complex with a canonical cell-complex structure which it inherits from that of the polyhedron

$$
P(d):=\left\{f \in \mathbb{R}^{X}: f(x)+f(y) \geq x y \text { for all } x, y \in X\right\}
$$

because $T(d)$ is easily seen to be nothing but the union of all compact faces of $P(d)$.

Next, one may also form the Buneman complex $B(d)$ that consists exactly of those maps $\mu$ from the set

$$
\mathcal{P}^{*}(X):=\{A \subseteq X: \emptyset \neq A \neq X\}
$$

of proper subsets of $X$ into $\mathbb{R}_{\geq 0}$ that satisfy the condition

(B1) $A, B \in \mathcal{P}^{*}(X), A \cup B=X$, and $\mu(A) \neq 0 \neq \mu(B)$ implies $A \cap B=\emptyset$, as well as

(B2) $A \in \mathcal{P}^{*}(X)$ implies $\mu(A)+\mu(X-A)=\alpha_{d}(A)$, where $\alpha_{d}(A)$ is defined to be the number

$$
\frac{1}{2} \min \left(\max \left(a b+a^{\prime} b^{\prime}, a b^{\prime}+a^{\prime} b, a a^{\prime}+b b^{\prime}\right)-a a^{\prime}-b b^{\prime}: a, a^{\prime} \in A ; b, b^{\prime} \in X-A\right) .
$$

One may observe that $X$ can also be mapped into $B(d)$ in a canonical fashion by associating, to each $x \in X$, the map

$$
\mu_{x}: \mathcal{P}^{*}(X) \rightarrow \mathbb{R}_{\geq 0}: A \mapsto \begin{cases}0 & \text { if } x \in A \\ \alpha_{d}(A) & \text { else, }\end{cases}
$$

that $B(d)$ can also be viewed as a polyhedral complex (contained in $\mathbb{R}^{\mathcal{P}^{*}(X)}$ ) that has a canonical cell-complex structure, too, because it consists of the union of faces of the affine hypercube

$$
H(d):=\left\{\mu \in \mathbb{R}_{\geq 0}^{\mathcal{P}^{*}(X)}: \mu \text { satisfies }(\mathrm{B} 2)\right\}
$$


and that it also carries a canonical metric defined by restricting the $l_{1}$-metric of $\mathbb{R}^{\mathcal{P}^{*}(X)}$ to $B(d)$ and scaling it by one-half:

$$
\left\|\mu_{1}, \mu_{2}\right\|_{1}:=\frac{1}{2} \sum_{A \in \mathcal{P}^{*}(X)}\left|\mu_{1}(A)-\mu_{2}(A)\right|
$$

$\left(\mu_{1}, \mu_{2} \in B(d)\right)$ so that

$$
\left\|\mu_{1}, \mu_{2}\right\|_{1}=\sum_{x \in A \in \mathcal{P}^{*}(X)}\left|\mu_{1}(A)-\mu_{2}(A)\right|
$$

holds for every $x \in X$.

One may finally observe that there exists a canonical linear (and, hence, affine) map $\Lambda$ from $\mathbb{R}^{\mathcal{P}^{*}(X)}$ into $\mathbb{R}^{X}$ defined by

$$
\Lambda: \mathbb{R}^{\mathcal{P}^{*}(X)} \rightarrow \mathbb{R}^{X}: \mu \mapsto\left(f_{\mu}: X \rightarrow \mathbb{R}: x \mapsto \sum_{x \in A \in \mathcal{P}^{*}(X)} \mu(A)\right) .
$$

One can now re-interpret certain well-known results obtained within the context of phylogenetic analysis as asserting that the restriction $\Lambda(d):=\left.\Lambda\right|_{B(d)}$ of the map $\Lambda$ to $B(d)$ induces a bijective and isometric map between $B(d)$ and $T(d)$ that is also a polyhedral-complex isomorphism provided that $T(d)$ (and hence also $B(d)$ ) is of dimension one, or-equivalently-an (R-)tree, or-still equivalently-that $d$ satisfies the so-called four-point condition

(B3) $x y+u v \leq \max (x u+y v, x v+y u)$ for all $x, y, u, v \in X$

(see [3], [5], and [7]).

Obviously, the main result of this paper implies that-more generally- $\Lambda(d)$ will always be a polyhedral-complex isomorphism provided that $\Lambda(d)$ is bijective. So, one may ask whether or not there are further metrics, not necessarily satisfying (B3), for which $\Lambda(d)$ is bijective. Amazingly enough, this question could be answered definitively in [10], and it follows from the characterization of those metrics given there in terms of certain relaxations of the four-point condition (B3) (see also [8] and [9] for related results) that this is the case for every set of cardinality at most four, while it holds for a set $X$ of cardinality five if and only if the metric $d$ is totally split decomposable (see [1]), and for a set of cardinality at least six if and only if $\Lambda\left(\left.d\right|_{Y \times Y}\right)$ is bijective for the restriction $\left.d\right|_{Y \times Y}$ of $d$ to every subset $Y$ of cardinality six in which case $\Lambda(d)\left(\mu_{x}\right)=h_{x}$ as well as $\left\|\mu_{x}, \mu_{y}\right\|_{1}=x y$ also holds for all $x, y \in X$.

Remarkably, $\Lambda(d)$ is not necessarily an isometry even if it is a polyhedral-complex isomorphism and, hence, induces, at least, an isometry

$$
\left\{\mu_{x}: x \in X\right\} \rightarrow\left\{h_{x}: x \in X\right\}
$$

between the images of $X$ in $B(d)$ and in $T(d)$, respectively. It is shown in [11] that $\Lambda(d)$ is indeed an isometry if it is a bijection provided $\# X \leq 5$ holds while, just as above, it is an isometry in case $\# X \geq 6$ if and only if $\Lambda\left(\left.d\right|_{Y \times Y}\right)$ is an isometry for all $Y \subseteq X$ with $\# Y=6$. Moreover, provided $\Lambda(d)$ is a bijection, this holds if and only if, for $A, B, C \in \mathcal{P}^{*}(X)$ with $\alpha_{d}(A), \alpha_{d}(B), \alpha_{d}(C)>0$ and

$$
\emptyset \neq A \cap B \neq A \neq A \cup B \neq X
$$


as well as

$$
\emptyset \neq A \cap C \neq A \neq A \cup C \neq X,
$$

one has $\emptyset=B \cap C$, or $B \cap C=B$, or $B=B \cup C$, or $B \cup C=X$, that is, if and only if the associated set system

$$
\left\{A \in \mathcal{P}^{*}(X): \alpha_{d}(A)>0\right\},
$$

is 3-cross-free according to the definition given by Karzanov in [20] (see also [14] and [18]).

\section{Acknowledgment}

We thank Günter Ziegler for his helpful comments regarding an earlier version of this paper.

\section{Appendix}

In this appendix we recall briefly how polyhedral complexes can be used to establish de Bruijn's famous dualization principle that allows the construction of zonotopal Penrosetype tilings from multigrids of hyperplanes: Consider a discrete family of hyperplane equations

$$
\left\{h_{i}\left(x_{1}, x_{2}, \ldots, x_{n}\right)=a_{i_{1}} x_{1}+a_{i_{2}} x_{2}+\cdots+a_{i_{n}} x_{n}+c_{i}\right\}_{i \in I},
$$

with $I$ denoting some index set. Further, assume (without loss of generality) that $c_{i} \leq 0$ holds for all $i$ in $I$, and note that this condition implies that our requirement that the above family of hyperplane equations be a discrete family is then equivalent to requiring that, for every point $P=\left(x_{1}, x_{2}, \ldots, x_{n}\right) \in \mathbb{R}^{n}$, there be only finitely many $i \in I$ with $h_{i}(P) \geq 0$, that is, that only finitely many hyperplanes $H_{i}:=\left\{P \in \mathbb{R}^{n}: h_{i}(P)=0\right\}$ $(i \in I)$ separate $(0,0, \ldots, 0)$ from $P$.

Clearly, there is a tiling of $\mathbb{R}^{n}$ canonically defined by such a family of hyperplanes, whose tiles consist exactly of all subsets $T$ of $\mathbb{R}^{n}$ that are of the form

$$
T=T(P):=\left\{Q \in \mathbb{R}^{n}: \operatorname{sgn}\left(h_{i}(Q)\right)=\operatorname{sgn}\left(h_{i}(P)\right) \text { or } h_{i}(Q)=0\right\}
$$

$\left(P \in \mathbb{R}^{n}\right)$. De Bruijn observed that there is a simple way to construct a (topological) dual of this tiling by associating, to each $P \in \mathbb{R}^{n}$, the subset

$$
\begin{aligned}
T^{*}(P):=\left\{\sum_{i \in I} \alpha_{i}\left(a_{i_{1}}, a_{i_{2}}, \ldots, a_{i_{n}}\right): \alpha_{i}:=0 \text { if } h_{i}(P) \leq 0,\right. \\
\left.\alpha_{i}:=1 \text { if } h_{i}(P) \geq 0, \text { and } \alpha_{i} \in[0,1] \text { if } h_{i}(P)=0\right\} .
\end{aligned}
$$

Moreover, it was further observed in [4] that this tiling consists exactly of the projections into (or, rather up to) $\mathbb{R}^{n}$ of those faces $F$ of the (possibly unbounded) zonotope $B$ in 
$\mathbb{R}^{n+1}$ defined by

$$
B:=\left\{\sum_{i \in I} \alpha_{i}\left(a_{i_{1}}, a_{i_{2}}, \ldots, a_{i_{n}}, c_{i}\right): \alpha_{i} \in[0,1] \text { almost all } \alpha_{i}=0\right\}
$$

that satisfy the condition

$$
\left(x_{1}, x_{2}, \ldots, x_{n}, x_{n+1}+\varepsilon\right) \notin B
$$

for every $\left(x_{1}, x_{2}, \ldots, x_{n}, x_{n+1}\right) \in F$ and every positive $\varepsilon$, that is, de Bruijn's tiling is exactly the projection of the polyhedral complex consisting of all faces $F$ that belong to the "upper boundary" of $B$ (relative to the last coordinate along which we project $\mathbb{R}^{n+1}$ onto $\mathbb{R}^{n}$ ).

\section{References}

1. H.-J. Bandelt and A. Dress, A canonical decomposition theory for metrics on a finite set, Adv. in Math. 92 (1992), 47-105.

2. H.-J. Bandelt and A. Dress, Split decomposition: a new and useful approach to phylogenetic analysis of distance data, Mol. Phylogen. Evol. 1(3) (1992), 242-252.

3. J. Barthélemy and A. Guenoche, Trees and Proximity Representations, Wiley, New York, 1991.

4. J. Bohne, A. Dress, and S. Fischer: A simple proof for de Bruijn's dualization principle, Sankhya: Indian J. Statist., special volume dedicated to the memory of R. C. Bose, 54 (1992), 74-84.

5. P. Buneman, The recovery of trees from measures of dissimilarity, in Mathematics in the Archaeological and Historical Sciences, pp. 387-395, F. Hodson et al., eds., Edinburgh University Press, Edinburgh, 1971.

6. J. Dopazo, A. Dress, and A. von Haeseler, Split decomposition: a technique to analyze viral evolution, PNAS 90 (1993), 10320-10324.

7. A. Dress, Trees, tight extensions of metric spaces, and the cohomological dimension of certain groups: a note on combinatorial properties of metric spaces, Adv. in Math. 53 (1984), 321-402.

8. A. Dress, K. Huber, and V. Moulton, Some variations on a theme by Buneman, Ann. Combin. 1 (1997), 339-352.

9. A. Dress, K. T. Huber, and V. Moulton, A comparison between two distinct continuous models in projective cluster theory: the median and the tight-span construction, Ann. Combin. 2 (1998), 299-311.

10. A. Dress, K. T. Huber, and V. Moulton, An explicit computation of the injective hull of certain finite metric spaces in terms of their associated Buneman complex, Preprint.

11. A. Dress, K. T. Huber, and V. Moulton, Split-decomposable metrics of combinatorial dimension two, Preprint.

12. A. Dress, K. T. Huber, and V. Moulton, Some new perspectives regarding sparse continuous models for discrete data structures, Electron. Notes Discrete Math. 2 (1998).

13. A. Dress, D. Huson, and V. Moulton, Analyzing and visualizing distance data using SplitsTree, Discrete Appl. Math. 71 (1996), 95-110.

14. A. Dress, J. Koolen, and V. Moulton, $4 n-10$, Preprint.

15. A. Dress, V. Moulton, and W. Terhalle, T-theory: an Overview, European J. Combin. 17 (1996), 161-175.

16. A. Dress and W. Terhalle, A combinatorial approach to p-adic geometry, part I: the process of completion, Geom. Dedicata 46 (1993), 127-148.

17. A. Dress and W. Terhalle, The tree of life and other affine buildings, Doc. Math. J. DMV Extra Volume ICM III (1998), 565-574.

18. T. Fleiner, The size of 3-cross-free families, Preprint.

19. J. Isbell, Six theorems about metric spaces, Comment. Math. Helv. 39 (1964), 65-74.

20. A. Karzanov, Combinatorial methods to solve cut-determined multiflow problems, in Combinatorial Methods for Flow Problems, No. 3, pp. 6-69, A. Karzanov, ed., Vsesoyuz. Nauchno-Issled. Inst. Sistem. Issled., Moscow, 1979 (in Russian). 
21. V. Klee and P. Kleinschmidt, Convex polytopes and related complexes, in Handbook of Combinatorics, Vol. 1, pp. 875-917, R. Graham et al., eds., North-Holland, Amsterdam, 1995.

22. P. Lockhart, A. Meyer, and D. Penny, Testing the phylogeny of swordtail fishes using split decomposition and spectral analysis, J. Mol. Evol. 41 (1995), 666-674.

23. A. Schrijver, Polyhedral combinatorics, in Handbook of Combinatorics, Vol. 1, pp. 1649-1704, R. Graham et al., eds., North-Holland, Amsterdam, 1995.

24. A. E. Taylor and D. C. Lay, Introduction to Functional Analysis, 2nd edn., Wiley, New York, 1980.

25. G. Ziegler, Lectures on Polytopes, Graduate Texts in Mathematics 152, Springer-Verlag, New York, 1995.

Received September 30, 1999, and in revised form February 25, 2000. Online publication May 8, 2000. 